\title{
Ischemia of the hand secondary to radial artery thrombosis: A report of three cases
}

\author{
Sachin A Chitte BSc, Karen Veltri MSc PhD, Achilleas Thoma MD MSc FRCS(C)
}

SA Chitte, K Veltri, A Thoma. Ischemia of the hand secondary to radial artery thrombosis: A report of three cases. Can J Plast Surg 2003;11(3):145-148.

Upper extremity arterial thrombosis, though rare, is more prevalent on the ulnar side of the circulation, with the most common etiology being repetitive blunt trauma to the hypothenar eminence. Radial artery thrombosis is even more rare and when it does occur, is associated most often with iatrogenic cannulation, producing subsequent thrombi and emboli.

Three cases of spontaneous thrombosis of the radial artery extending to the superficial palmar arch and the princeps pollicis artery are presented here. Two patients underwent thrombectomies and one underwent excision of the thrombosed segment and reconstruction with a reversed saphenous vein graft. The etiology of the thrombus was consistent with cancer in two cases and trauma in one.

Key Words: Etiology; Ischemia; Pathophysiology; Radial artery; Thrombosis

\section{L'ischémie de la main secondaire à la throm- bose de l'artère radiale : Rapport de trois cas}

La thrombose artérielle des extrémités supérieures, bien qu'elle soit rare, est plus prévalentes du côté cubital de la circulation, l'étiologie la plus courante étant un traumatisme contondant à l'éminence hypothénar. La thrombose de l'artère radiale est encore plus rare et, lorsqu'elle se produit, elle s'associe surtout à une cannulation iatrogène, qui provoque une thrombose et une embolie subséquentes.

Trois cas de thrombose spontanée de l'artère radiale s'étendant jusqu'à l'arche palmaire et à l'artère princeps pollicis sont présentés. Deux patients ont subi une thrombectomie et un a subi une excision du segment thrombosé et une reconstruction à l'aide d'une greffe de la veine saphène renversée. L'étiologie de la thrombose était compatible avec un cancer dans deux cas, et avec un traumatisme dans le troisième cas.
T ower extremity thrombosis has been estimated to be six Ltimes more common than upper extremity thrombosis (1). When upper extremity thrombosis does occur, it is frequently seen in the ulnar artery superficial palmar arch and proper digital arteries with relative sparing of the radial artery and common digital arteries (2). Upper-extremity emboli comprise only $15 \%$ to $20 \%$ of all emboli and of these, $70 \%$ are cardiac and the remaining $30 \%$ are subclavian in origin (3). Cardiac emboli often occur after myocardial infarction, originating from ventricular aneurysms and atrial fibrillation. These emboli tend to lodge at the brachial artery bifurcation although smaller and 'shower emboli' may lodge more distally $(2,3)$. In the absence of trauma or emboli, the vascular symptoms of upper extremity thrombosis comprise more widespread systemic disease.

Ulnar artery thrombosis occurs secondary to single or repeated blunt trauma to the hypothenar eminence and is known as 'hypothenar hammer syndrome' (3).

Radial artery thrombosis, though extremely rare, has been reported in its deep branch where the artery crosses the dorsal ridge of the trapezium and from just proximal to the origin of the princeps pollicis artery to just distal to the superficial palmar branch of the radial artery $(1,2,4-8)$. Thrombosis may arise due to a wide variety of causes including iatrogenic cannulation, emboli, atherosclerosis or blunt and penetrating trauma $(4,5,9)$. Cannulation of the radial artery results in endothelial injury, thus, exposing subendothelial collagen leading to platelet adherence and subsequent thrombus formation (3). A well-documented cause of radial artery thrombosis occurs secondary to radial artery cannulation (2). One study has shown this to occur in up to $60 \%$ of cases (9), necessitating microvascular consultation.

Occlusion of the radial artery and superficial palmar arch or occlusion of the radial artery alone with an incomplete palmar arch leads to ischemia (9). In the latter, the thrombus extends to the common digital arteries. In turn, increased sympathetic discharge from a thrombosed distal radial or ulnar artery causes secondary vasospasm of collateral circulation leading to ischemia (3).

A unique 'hyperextension-compression' hypothesis in bodybuilders performing bench presses has also been implicated in radial artery thrombosis (5). External compression of the radial artery may also lead to sludging of the blood and subsequent thrombosis. McNamara et al (4) showed that in four of nine patients who suffered nontraumatic radial artery thrombosis, the radial artery was 'caught' between the extensor pollicis longus tendon and the trapeziometacarpal joint. Ganglionic cysts have also been implicated as sources of compression (3).

Arterial emboli almost always originate at the thoracic outlet secondary to subclavian artery compression (due to prolonged crutch use) contributing to an axillary artery aneurysm and 'thoracic outlet syndrome' (3).

Angiography is the diagnostic test of choice. It accurately localizes the level of occlusion and permits more effective

Department of Surgery, Division of Plastic and Reconstructive Surgery, St Joseph's Healthcare; McMaster University, Hamilton, Ontario Correspondence: Dr Achilleas Thoma, 206 James Street. South, Suite 101, Hamilton, Ontario L8P 3A9. Telephone 905-523-0019,

fax 905-523-0229, e-mail athoma@mcmaster.ca 


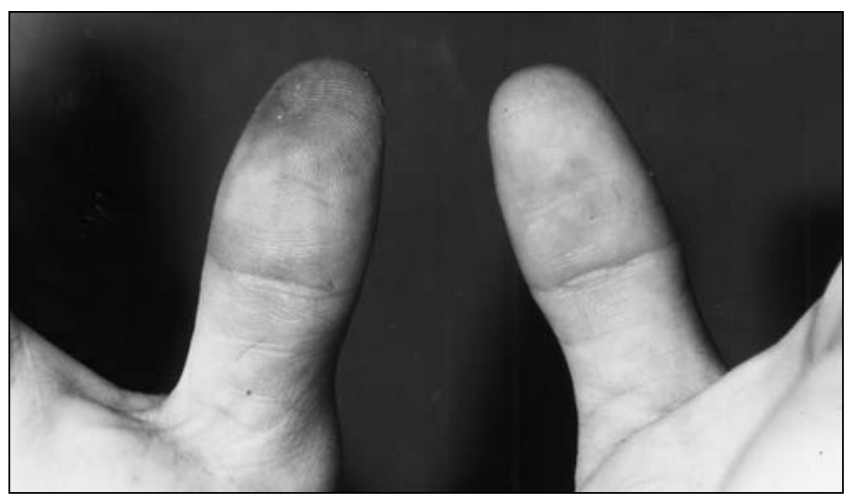

Figure 1) Right thumb showing discolouration as compared with opposite thumb

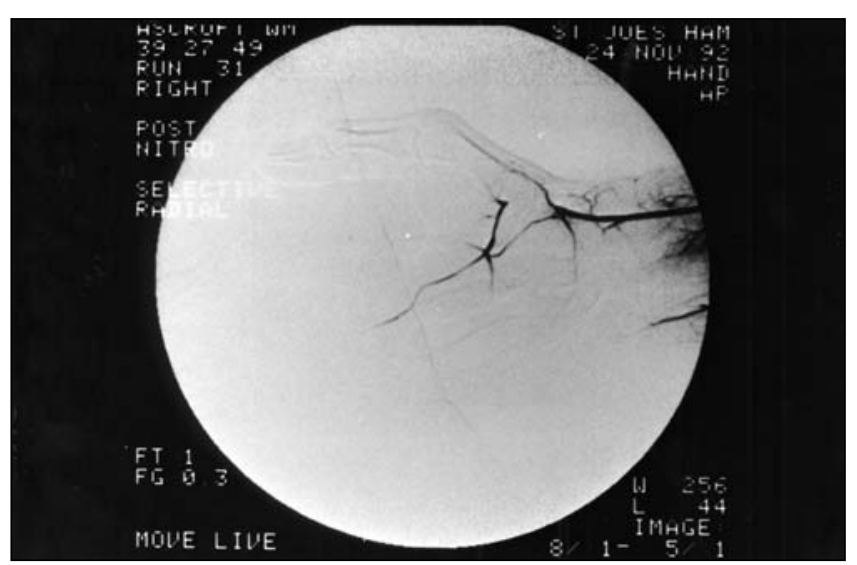

Figure 2) Angiogram showing occlusion of the radial artery extending from the anatomical 'snuffbox' to the bifurcation of the princeps pollicis artery and the palmar arch

patient management. Initial conservative management involves discontinuing the offending activity, avoiding cold environments and cessation of smoking. Medical interventions include the use of vasodilators, steroids, chemical sympathectomy and stellate ganglion block. Thrombolytic therapy with streptokinase administered within $36 \mathrm{~h}$ of presentation has been shown to be successful in four of six cases (10). Surgical interventions include periarterial sympathectomy to relieve vasospasm, and thrombus excision, ligation and resection with microvascular anastomosis. Additional surgical options include thrombectomy and excision with arterial reconstruction using an interpositional vein graft to re-establish pulsatile flow (11).

Three cases of spontaneous radial artery thrombosis are presented here.

\section{Case 1}

\section{CASE PRESENTATION}

A 27-year-old male production manager presented to the emergency room (ER) with numbness in the median nerve distribution and discolouration of the right thumb and index finger for a duration of four days. His right thumb and index finger were cold and blue with cutaneous temperatures of $27.5^{\circ} \mathrm{C}$ and $28.4^{\circ} \mathrm{C}$, respectively (Figure 1 ). He was a nonsmoker and otherwise healthy. The attending physician immediately prescribed acetylsalicylic acid (650 mg/day). An

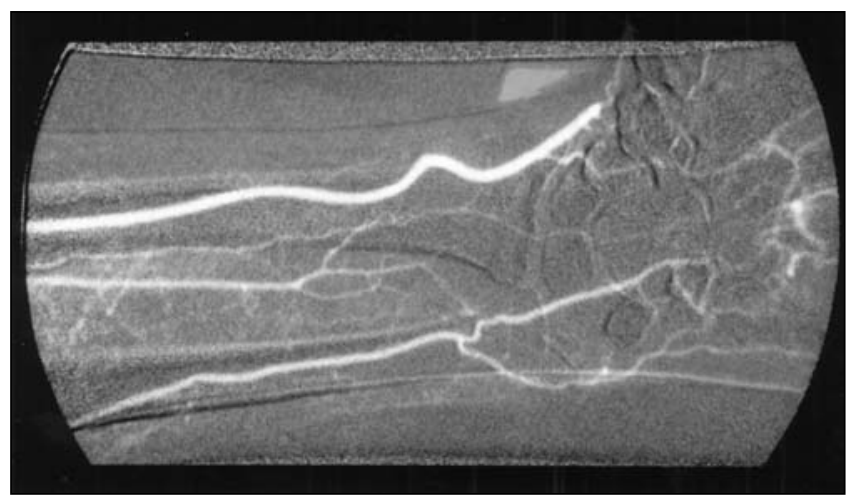

Figure 3) Angiogram showing occlusion of the radial artery extending from the anatomical 'snuffbox' to the palmar arch

angiogram showed occlusion of the radial artery at the anatomical 'snuffbox' with partial refilling of the digital arteries by the deep palmar arch and the ulnar artery (Figure 2). At surgery, a thrombus was found in the radial artery at the anatomical snuffbox extending to the bifurcation of the princeps pollicis artery and the palmar arch. A $3 \mathrm{~cm}$ segment of the thrombosed artery was resected and the defect was bridged with a reversed saphenous vein graft. Pathological examination confirmed this to be an organized thrombus. No exact cause for the thrombus was determined. Of note, the patient reported banging the dorsum of his right hand with a piece of metal at work a few days before his presentation. However, there was no fracture, laceration or bruising of the hand to suggest any significant injury to the hand. Postoperatively, his symptoms improved and his thumb was salvaged.

\section{Case 2}

A 65-year-old man presented to the ER with a sudden onset of pain, tingling, mottling and coolness of the left hand that began the previous night. He was a smoker who had a right retromolar trigone squamous cell carcinoma (SCC) resected and the defect was reconstructed with a right osteocutaneous free fibular flap 25 days before presentation. A fibular flap was used instead of a radial forearm flap due to a bilaterally positive Allen's test. An angiogram showed an abrupt occlusion in the left radial artery at the anatomical snuffbox (Figure 3). At surgery, a thrombus was found in the radial artery extending from the anatomical snuffbox to the bifurcation of the princeps pollicis artery and the superficial palmar arch (Figure 4). The thrombus was removed with a $2 \mathrm{~mm}$ Fogarty catheter inserted downstream, $10 \mathrm{~cm}$ into the palmar arch. Pathological examination revealed a $7.6 \mathrm{~cm}$ recent thrombus (Figure 5). At no time during his previous head and neck procedures was his radial artery cannulated. Two months after the radial artery thrombectomy, he was found to have multiple cutaneous SCC metastases to his left palm. At autopsy 2.5 months later, he was found to have SCC metastases to the myocardium as well as a malignant left ventricular mural thrombus.

Case 3

A 52-year-old woman presented in the ER with gradual onset of abnormal sensation and ischemic pain in her left wrist and hand lasting four days. At presentation, she showed bluish discolouration and frank necrosis of the left index and long finger 


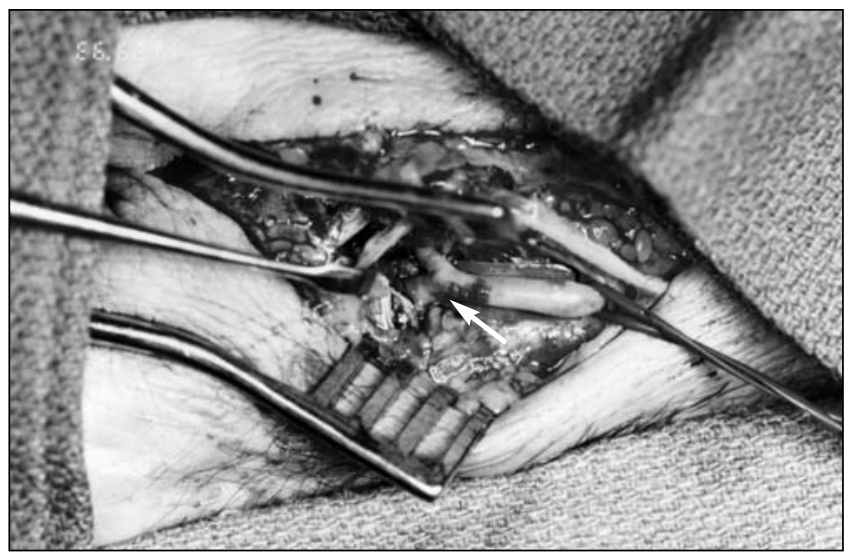

Figure 4) Occlusion of the radial artery extending to the bifurcation of the princeps pollicis artery and the superficial palmar arch. (Arrow indicates bifurcation)

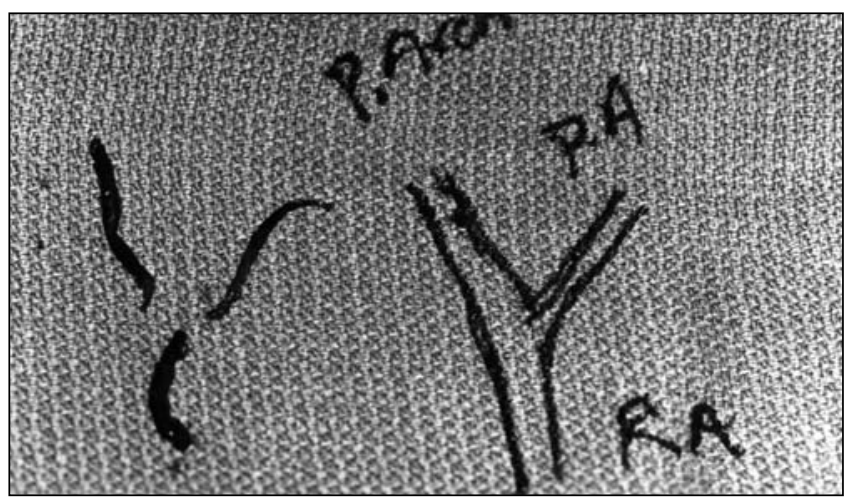

Figure 5) At left is a 'saddle-shaped' thrombus that was removed at the level of the bifurcation. It is diagrammatically represented at right. PA Princeps artery; P Arch Palmar arch; RA Radial artery

tips (Figure 6). She was discharged home with analgesics and follow-up plans. However over the following two days the ischemia progressed to a degree that pain control required hospitalization. Her left hand was cooler than the right and the radial side of her left hand was cooler than the ulnar side. Her past medical history included smoking, deQuervain's tenosynovitis in her left hand and cancer of the left breast $\left(\mathrm{T}_{1} \mathrm{~N}_{0} \mathrm{M}_{0}\right)$ treated by mastectomy. Five months earlier, she was diagnosed with a stage 3 carcinoma of the caecum requiring hemicolectomy. She was treated with radiotherapy and was taking several chemotheraputic agents, including the immune stimulant levamisole. An angiogram showed a complete occlusion of the left radial artery at the level of the anatomical snuffbox. At surgery, a thrombus was found beginning $3 \mathrm{~cm}$ proximal to the wrist crease. The thrombus was removed by arteriotomy at the anatomical snuffbox and using a $2 \mathrm{~mm}$ Fogarty catheter which was advanced $5 \mathrm{~cm}$ into the artery distally (Figure 7). The saddle configuration of the thrombus suggested involvement of the princeps pollicis artery as it originated from the palmar arch. Due to poor improvement of symptoms, she required a left thoracic sympathectomy five days later. The next day, a repeat angiogram showed left axillary artery embolization that required an

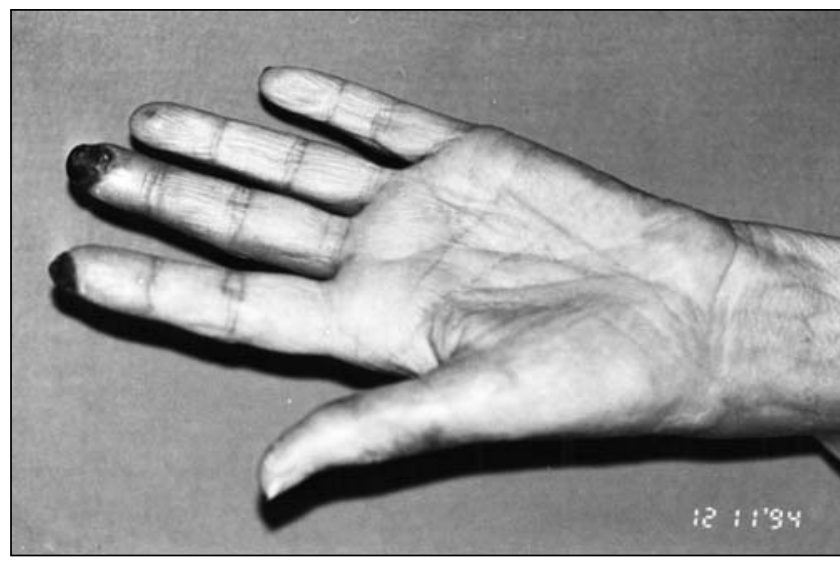

Figure 6) Necrotic index and long finger tips due to radial artery thrombosis

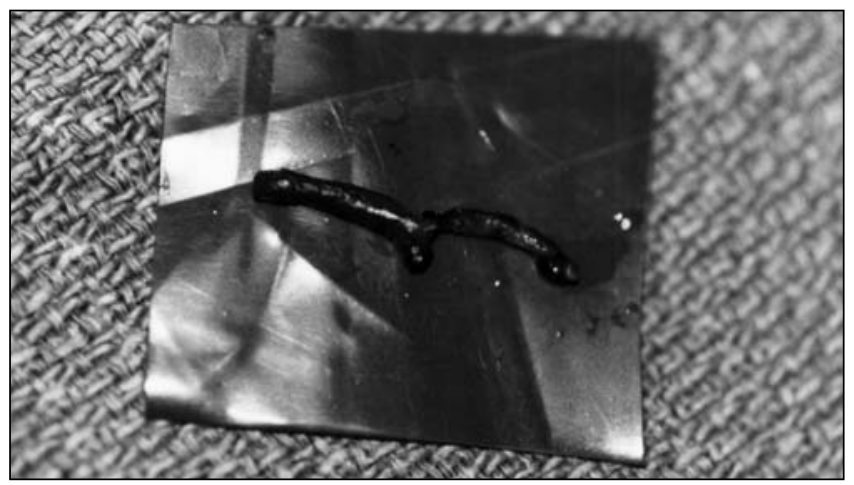

Figure 7) Saddle-shaped thrombus removed from the bifurcation of the princeps artery and the palmar arch

embolectomy more proximally. One month later she required an additional right femoral artery embolectomy which was unsuccessful culminating in a below-knee amputation of the right leg. She died three months after the initial presentation for hand symptoms. At the time of the initial hand presentation, she had no history of radial artery cannulation. It was thought initially that levamisole may have been responsible for the recurrent embolism via hyperstimulation of the immune system. Autopsy results, however, showed marantic endocarditis which was clearly the cause of the embolus formation.

\section{DISCUSSION}

Arterial occlusive disease in the upper extremity can be caused by local arterial trauma, emboli or systemic disease (3).

Direct trauma is a well-documented cause of radial artery thrombosis among baseball players and workers who frequently receive impacts at their thenar eminences $(4,8)$. It is possible that Case 1, an otherwise healthy patient, fits this category because he reportedly banged the dorsum of his hand with a piece of metal.

Cases 2 and 3 appeared to have occluded radial arteries due to malignancy and cardiac ventricular thrombus. 
Sludging of blood in low flow states has been implicated as a cause of thrombosis and embolism. Sludging can occur after intra-arterial drug injections, polycythemia, meningococcal septicemia and malignant disease (2) and, in our patients with previous malignancies (Case 2, Case 3 ), this may have also contributed to the occlusion.

Among the well-described causes of hypercoagulability leading to thrombosis, direct and paraneoplastic effects of malignancy play a significant role. These are commonly associated with lung, gastrointestinal, breast and ovarian cancer as well as lymphoma (12). A previous breast malignancy as well as cecum cancer in Case 3, may have contributed to the hypercoagulability and subsequent radial artery occlusion.

The usual vascular configuration of the hand is characterized by the dominant blood supply of the ulnar artery (Figure 8 ). The superficial branches of the ulnar and radial arteries join to form the superficial palmar arch which, in turn, gives rise to the common palmar digital arteries. This arch is complete in $80 \%$ of cases, but significant variation does exist (9). The deep palmar arch is formed by the deep branch of the radial artery as it joins the deep branch of the ulnar artery. The thumb and the radial side of the index finger are supplied by the princeps pollicis and radialis indicis arteries, respectively. The origin of these arteries is variable; they may originate off the deep palmar arch but more commonly branch off of the superficial palmar arch. Both arteries run parallel to the first metacarpal bone before dividing at the level of the metacarpophalangeal joint to form the digital arteries to the thumb and the index finger $(6,7,9)$.

When harvesting the commonly used radial forearm free flap, the radial artery is completely sacrificed, yet ischemic symptoms do not usually occur. However, two of the patients in the present study developed ischemic symptoms with a thrombosed radial artery in the presence of an intact palmar arch. Why this discrepancy? The answer lies in the anatomical level of the occlusion. A critical part of the palmar arch must remain patent to allow backflow from the ulnar artery. This critical part is the bifurcation between the palmar arch and princeps pollicis artery (Figure 8, grey region). This portion remains patent during harvesting of the radial free forearm flap; however, this was the exact level of occlusion in our patients.

\section{REFERENCES}

1. Wirsing P, Andriopoulos A, Botticher R. Arterial embolectomies in the upper extremity after acute occlusion. J Cardiovasc Surg 1983;24:40-2.

2. Jones NF. Acute and chronic ischemia of the hand: Pathophysiology, treatment and prognosis. J Hand Surg (Am) 1991;16:1074-83.

3. Zimmerman NB. Occlusive vascular disorders of the upper extremity. Hand Clinics 1993;9:139-50.

4. McNamara MG, Butler TE, Sanders WE, Pederson WC. Ischemia of the index finger and thumb secondary to thrombosis of the radial artery in the antomical snuffbox. J Hand Surg (Br) 1998;23:28-32.

5. Isenberg JS. Spontaneous exercise-induced thrombosis of the radial artery: A case report and literature review. Ann Plast Surg 2000;44:79-81.

6. Ferner H, ed. Penkopf Atlas of Topographical and Applied Human

\section{Vascular Supply--Right Hand A-P View}

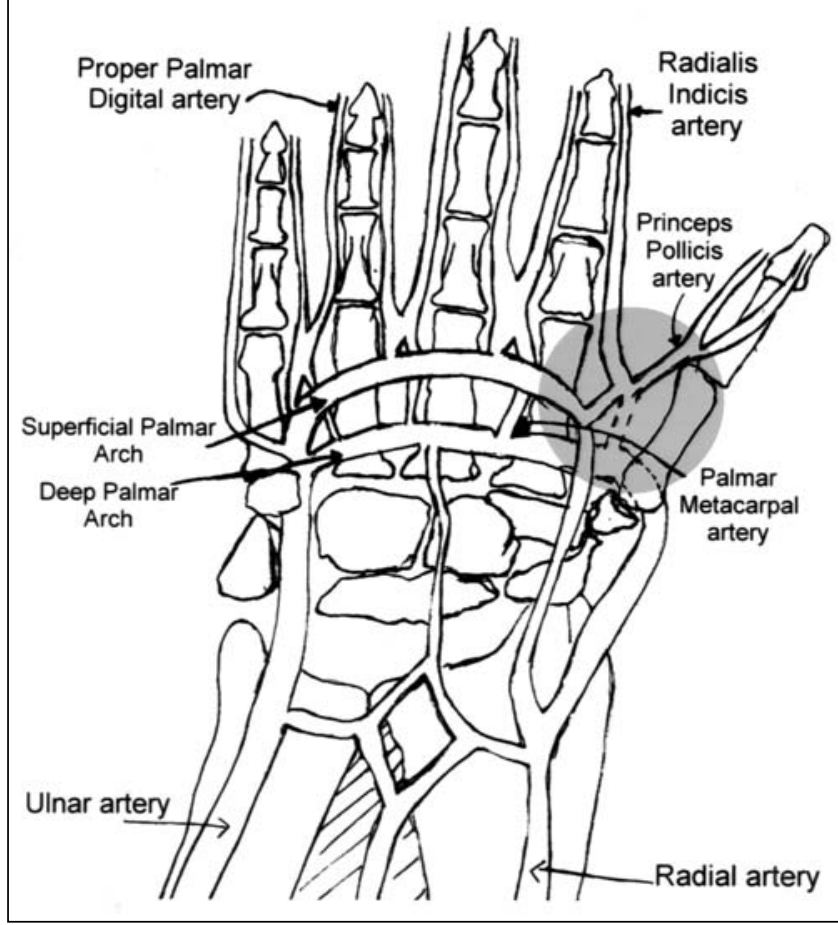

Figure 8) Diagrammatic representation of the vascular supply of the right hand. Note the birfurcation between the palmar arch and the princeps pollicis artery (grey region). A-P Anteroposterior view

\section{CONCLUSION}

In conclusion, when one is faced with an uncommon spontaneous radial artery occlusion, malignancy and cardiac embolization should be seriously considered. The thrombosis occurs at the critical junction of the princeps pollicis artery from the superficial palmar arch.

Anatomy. Volume II: Thorax, Abdomen and Extremities, 2nd edn Baltimore: Urban and Schwartzenberg, 1980.

7. Viegas SF. Hand Surgery Study Guide. New York: Springer-Verlag Inc, 1996.

8. Topper SM, Berger RA. Radial artery thrombosis in a young athlete: A case report. Am J Sports Med 1998;26:297-9.

9. Lee KL, Miller JG, Laitung G. Hand ischemia following radial artery cannulation. J Hand Surg (Br) 1995;20:493-5.

10. Jelalian C, Mehrhof A, Cohen IK, Richardson J, Merritt WH. Streptokinase in the treatment of acute arterial occlusion of the hand. J Hand Surg 1985;10:534-8.

11. Peris MD, Tomaino MM. Ulnar artery thrombosis: Evaluation and indications for operative treatment and surgical technique. Am J Orthop 1996;25:685-9.

12. Fauci AS, Brunwald E, Isselbacher KJ, et al, eds. Harrison's Principles of Internal Medicine. New York: McGraw Hill, 1998. 